\title{
Extracting Thickness and Tilt From 4D-STEM Datasets to Model the Influence on ABF Images
}

\author{
Abinash Kumar ${ }^{1}$, Celesta S. Chang ${ }^{2}$, Everett D. Grimley ${ }^{1},{\text { Zhen } \text { Chen }^{3} \text { and James M. LeBeau }}^{1}$ \\ 1. Department of Materials Science and Engineering, North Carolina State University, Raleigh, NC. \\ 2. Department of Physics, Cornell University, Ithaca, NY. \\ 3. School of Applied and Engineering Physics, Cornell University, Ithaca, NY.
}

Thin film epitaxial strain plays an important role in controlling the behavior of materials, including electrical/magnetic properties and phase transitions. Further, epitaxial strain can mediate properties by inducing lattice distortions, such as octahedral rotations, at film/substrate interfaces [1]. Developing an understanding of these distortions is thus essential to control film properties for a given system. One method to characterization this distortion is position averaged convergent beam electron diffraction (PACBED), which is also used to precisely quantify sample thickness, orientation, tilt, and even polarization $[2,3]$. With the introduction of 4D-STEM detectors, such as the EMPAD, PACBED patterns can be reconstructed after acquisition to map sample by averaging diffraction patterns over selected regions [4].

In this presentation, we analyze a 4D-STEM dataset of an epitaxial $\mathrm{Ca}_{2} \mathrm{RuO}_{4}$ thin film. PACBED patterns extracted in unit cell increments along the growth direction are shown in Figure 1. To determine sample thickness and sample tilt, the patterns are matched to frozen phonon multislice simulations by least squares fitting. The simulated patterns have been resized and rotated for comparison to the experimental patterns. Figure 2 shows the matching simulated patterns to within $\pm 1 \mathrm{~nm}$ thickness and \pm 1 mrad tilt. Simulated using these measurements, ABF images are shown in Figure 3. It is observed that for relatively minor tilts, large differences in the image occur. Specifically, the Ru atom columns are observed to shift relative to $\mathrm{Ca}$ and $\mathrm{O}$. These results highlight the need to measure tilt and thickness independent of ABF images.

Finally, we will also discuss approaches to automate sample tilt/thickness refinement by combining convolutional neural networks with least squares fitting to increase precision while reducing computation time [5].

References:

[1] S. J. May et al, Physical Review B 82 (2010), p. 014110.

[2] J. M. LeBeau et al, Ultramicroscopy 110 (2010), p. 118.

[3] J. Hwang et al, Applied Physics Letters 100 (2012), p. 191909.

[4] M. W. Tate et al, Microscopy and Microanalysis 22 (2016), p. 237.

[5] We would like to thank D. A. Muller for supplying the EMPAD data acquired from a thin film grown by H. Nair and D. G. Schlom. This material is based upon work supported by the National Science Foundation, as part of the Center for Dielectrics and Piezoelectrics under Grant Nos. IIP-1361571 and IIP-1361503. 

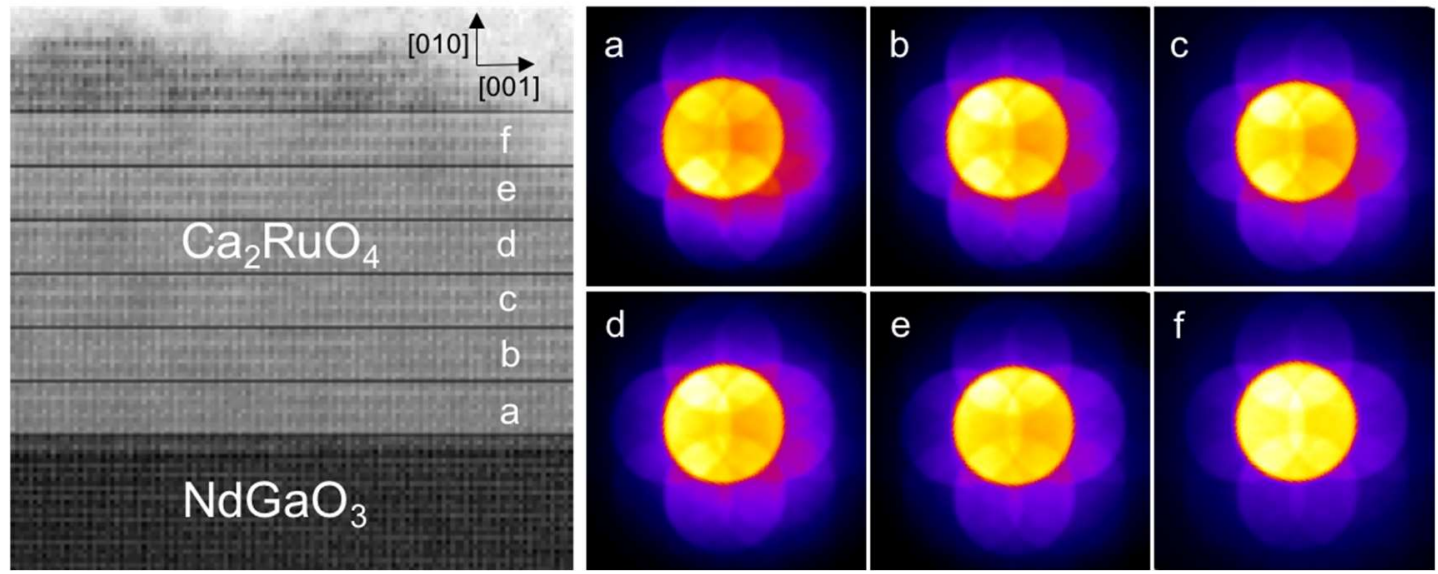

Figure 1. Experimental PACBED patterns extracted from the 4D-STEM dataset of a $\mathrm{Ca}_{2} \mathrm{RuO}_{4}$ thin film on $\mathrm{NdGaO}_{3}$. The convergence semi-angle is $7.9 \mathrm{mrad}$.

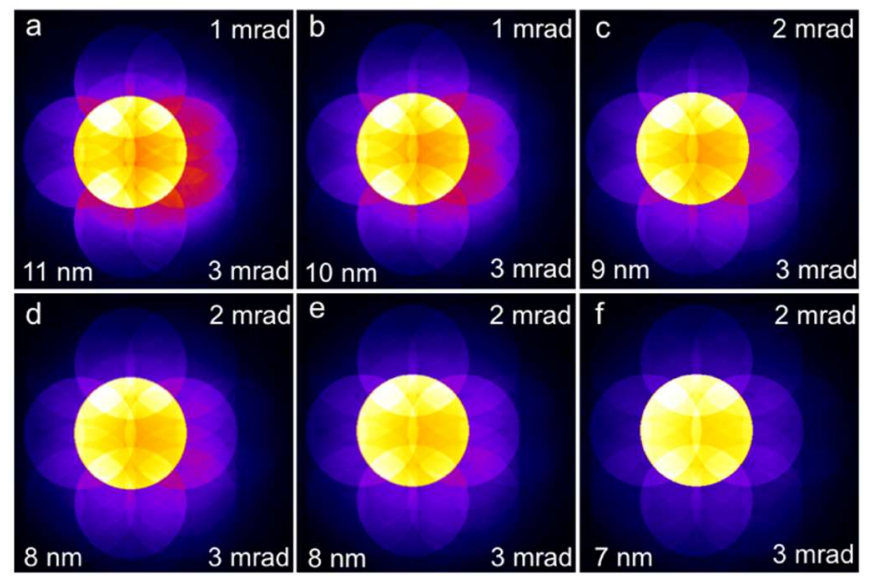

Figure 2. Simulated PACBED patterns (along [100]) fits to the experimental patterns in Figure 1 using a least squares method. (Top right - tilt value along [001], bottom left - thickness value, and bottom right tilt value along [010])

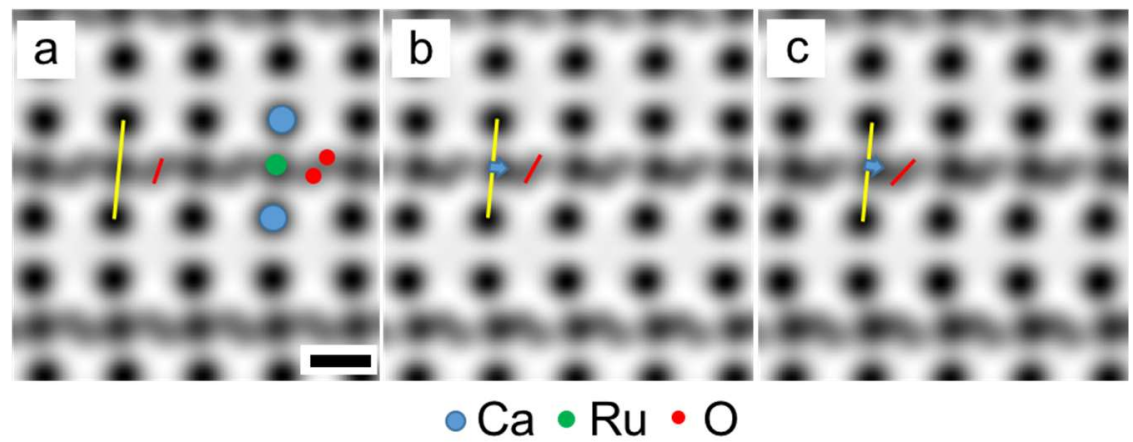

Figure 3. Simulated $A B F$ for $10 \mathrm{~nm}$ thick $\mathrm{Ca}_{2} \mathrm{RuO}_{4}$ with different sample tilt parameters, (a) without tilt, (b) $1 \mathrm{mrad}$ along [001] and $3 \mathrm{mrad}$ along [010] and (c) $2 \mathrm{mrad}$ along [001] and $3 \mathrm{mrad}$ along [010] respectively. (Scale bar - $2 \AA$ ) 